\title{
Evaluation of four colourimetric susceptibility tests for the rapid detection of multidrug-resistant Mycobacterium tuberculosis isolates
}

\author{
Ahmet Yilmaz Coban ${ }^{1 /+}$, Ahmet Ugur Akbal', Meltem Uzun², Belma Durupinar ${ }^{1}$ \\ ${ }^{1}$ Ondokuz Mayis University, Medical School Samsun, Department of Medical Microbiology, Samsun, Turkey \\ ${ }^{2}$ Istanbul University, Istanbul Medical School, Department of Medical Microbiology, Istanbul, Turkey
}

\begin{abstract}
The purpose of this study is to evaluate four rapid colourimetric methods, including the resazurin microtitre assay (REMA), malachite green decolourisation assay (MGDA), microplate nitrate reductase assay (MNRA) and crystal violet decolourisation assay (CVDA), for the rapid detection of multidrug-resistant (MDR) tuberculosis. Fifty Mycobacterium tuberculosis isolates were used in this study. Eighteen isolates were MDR, two isolates were only resistant to isoniazid (INH) and the remaining isolates were susceptible to both INH and rifampicin (RIF). INH and RIF were tested in $0.25 \mu \mathrm{g} / \mathrm{mL}$ and $0.5 \mu \mathrm{g} / \mathrm{mL}$, respectively. The agar proportion method was used as a reference method. MNRA and REMA were performed with some modifications. MGDA and CVDA were performed as defined in the literature. The agreements of the MNRA for INH and RIF were $96 \%$ and $94 \%$, respectively, while the agreement of the other assays for INH and RIF were 98\%. In this study, while the specificities of the REMA, MGDA and CVDA were $100 \%$, the specificity of the MNRA was lower than the others $(93.3 \%$ for INH and $90.9 \%$ for RIF). In addition, while the sensitivity of the MNRA was $100 \%$, the sensitivities of the others were lower than that of the MNRA (from 94.1-95\%). The results were reported on the seventh-10th day of the incubation. All methods are reliable, easy to perform, inexpensive and easy to evaluate and do not require special equipment.
\end{abstract}

Key words: resazurin microtitre assay - malachite green decolourisation assay - microplate nitrate reductase assay crystal violet decolourisation assay - multidrug-resistant tuberculosis

Mycobacterium tuberculosis is the oldest known infectious agent and remains a major public health problem (WHO 2014). An early detection of multidrug-resistant tuberculosis (MDR-TB) isolates allows for an appropriate and timely treatment (Martin et al. 2008). The proportion method, which is performed on Löwenstein-Jensen (LJ) and Middlebrook 7H10 or 7H11 agar media, is recommended as a reference method. However, this method requires at least three-six weeks to obtain results (Kent \& Kubica 1985). There are also rapid automated systems for drug susceptibility testing, but these systems are expensive and require equipment (Bwanga et al. 2009, Friedrich et al. 2011, Chang et al. 2012). Recently, many colourimetric methods, including the resazurin microtitre assay (REMA), or resazurin tube assay, malachite green decolourisation assay (MGDA), nitrate reductase assay (NRA) and crystal violet decolourisation assay (CVDA), have been developed. These methods are rapid, inexpensive, reliable and reproducible (Angeby et al. 2002, Coban et al. 2004, Martin et al. 2007, 2011, Palomino et al. 2007).

This paper aims to evaluate four rapid colourimetric methods, including the REMA, microplate NRA (MNRA), CVDA and MGDA methods, for the rapid de-

doi: 10.1590/0074-02760150136

+ Corresponding author: cobanay2003@gmail.com

Received 2 April 2015

Accepted 26 June 2015 tection of MDR-TB. In this study, these methods were compared with each other for the first time in terms of their sensitivity, specificity, positive predictive value (PPV) and negative predictive value (NPV) for isoniazid (INH) and rifampicin (RIF).

\section{MATERIALS AND METHODS}

Bacterial isolates - Fifty M. tuberculosis isolates were used in this study. Isolates were obtained from the Istanbul Faculty of Medicine and Samsun Chest Diseases and Thoracic Surgery Hospital. Eighteen isolates were MDR, two isolates were only resistant to INH and the remaining isolates were susceptible to both INH and RIF. All isolates from stocks were subcultured on $\mathrm{LJ}$ medium and freshly subcultured isolates were used in the study.

Chemical substances - Resazurin salt powder, sulfanilamide, n-1-naphthylethylenediamine dihydrochloride, crystal violet (CV) dye, INH and RIF were purchased from Sigma-Aldrich (Germany). Malachite green (MG) dye was purchased from Merck (Germany).

Preparation of chemical substances and reagents Resazurin powder was dissolved in sterile distilled water to a final concentration of $0.02 \%$, sterilised by filtration and stored at $4^{\circ} \mathrm{C}$ until use. Griess reagent consisted of one part $50 \%$ (vol $/ \mathrm{vol})$ concentrated hydrochloric acid, two parts $0.2 \%(\mathrm{wt} / \mathrm{vol})$ sulfanilamide and two parts $0.1 \%(\mathrm{wt} /$ vol) n-1-naphthylethylenediamine dihydrochloride. MG dye was dissolved in distilled water to a final concentration of $50 \mu \mathrm{g} / \mathrm{mL}$. CV dye was prepared in distilled water to a final concentration of $25 \mu \mathrm{g} / \mathrm{mL}$. All of the chemical substances and reagents were stored at $4^{\circ} \mathrm{C}$ until use. 


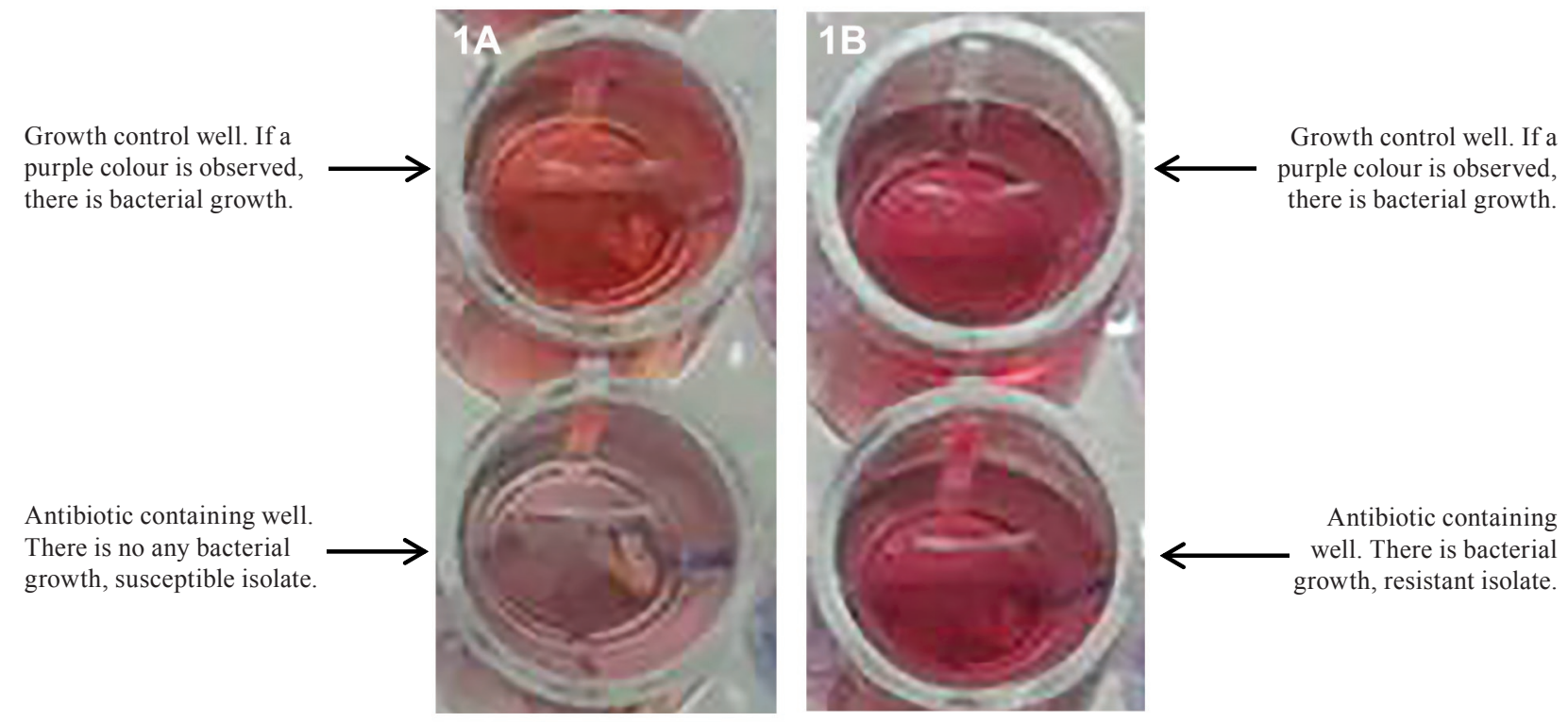

Fig. 1: microtitre nitrate reductase assay.

Preparation of media - The REMA, MNRA, CVDA and MGDA susceptibility tests were performed in Middlebrook $7 \mathrm{H} 9 \mathrm{~S}$ broth containing $0.1 \%$ casitone, $0.5 \%$ glycerol and $10 \%$ OADC. Middlebrook 7H9S broth with $1,000 \mu \mathrm{g} /$ $\mathrm{mL}$ potassium nitrate $\left(\mathrm{KNO}_{3}\right)$ was used for the MNRA.

Preparation of bacterial inoculum - Bacterial colonies that had been freshly grown on LJ media were transferred into tubes containing $5 \mathrm{~mL}$ saline solution and 15-20 glass beads and were vortexed for 1-2 min. The tube was kept in a vertical position for $30 \mathrm{~min}$ at room temperature to allow aerosols and large particles to precipitate. The turbidity of the supernatant was adjusted to that of the McFarland 1 standard. The prepared bacterial suspension was then diluted at an 1:10 ratio in Middlebrook 7H9S broth.

Preparation of antibiotics - All tests were performed in 96-well microtitre plates. One hundred microlitres of Middlebrook 7H9S broth (containing 0.1\% casitone, $0.5 \%$ glycerol and $10 \%$ OADC) were added into each well of the plates for the CVDA, MGDA and REMA methods. One hundred microlitres of Middlebrook $7 \mathrm{H} 9 \mathrm{~S}$ broth with $1,000 \mu \mathrm{g} / \mathrm{mL} \mathrm{KNO}_{3}$ were added into each well of the plate for the MNRA method. For each isolate, three wells containing INH, RIF and media without antibiotics were prepared. All plates were kept at $-20^{\circ} \mathrm{C}$ for two weeks.

Performing susceptibility tests - The MNRA and REMA were performed with some modifications. Briefly, the MNRA and REMA were performed using a critical concentration of drugs and in liquid media. The MGDA and CVDA were performed as defined in the literature. Each bacterial isolate was tested in each of the three wells containing INH $(0.25 \mu \mathrm{g} / \mathrm{mL})$, RIF $(0.5 \mu \mathrm{g} /$ $\mathrm{mL}$ ) and an antibiotic-free growth control for each of the susceptibility test methods. One hundred microlitres of bacterial suspension was inoculated into each well of the plates. After bacterial inoculation, all plates were incubated at $37^{\circ} \mathrm{C}$ under normal atmospheric conditions.

Evaluation of the MNRA method - After seven days of incubation, $50 \mu \mathrm{L}$ of Griess reagent was added into each of the growth control wells for the MNRA. If a purple colour was observed, Griess reagent was also added into the test wells. If a purple colour was observed in the test wells containing antibiotics, the bacterial isolate was considered to be resistant to the antibiotic that was being tested. The MNRA wells were evaluated for colour change within 3-5 $\mathrm{s}$ after the addition of the Griess reagent (Fig. 1A, B).

Evaluation of the REMA method - After seven days of incubation, $30 \mu \mathrm{L}$ of resazurin solution was added into each well with and without antibiotics. After the reagents were added into the wells, the plates were reincubated for one-three days under the same conditions. If the blue colour of resazurin changed to pink in the growth control well as in the wells containing antibiotics, the bacterial isolate was reported to be resistant to the antibiotics that were being tested (Fig. 2A, B).

Evaluation of the MGDA method - After seven days of incubation, $30 \mu \mathrm{L}$ of MG was added into each of the test wells and the plates were incubated for an additional one-three days. After the MG decolourised in the growth control well, the test wells containing antibiotics were evaluated for a colour change. If the MG decolourised as a result of bacterial growth in the wells containing antibiotics, these bacterial isolates were reported to be resistant to the antibiotics (Fig. 3A, B).

Evaluation of the CVDA method - On the seventh day of incubation, $25 \mu \mathrm{L}$ of $\mathrm{CV}$ was added into the wells and incubated for an additional one-three days. As the blue/ purple colour of $\mathrm{CV}$ was decolourised by the growth of bacteria, these bacterial isolates were considered to be resistant to that drug (Fig. 4A, B). 
Growth control well. If blue colour of resazurin changes to pink, there is bacterial growth.

Antibiotic containing well. There is no any bacterial growth, susceptible isolate.
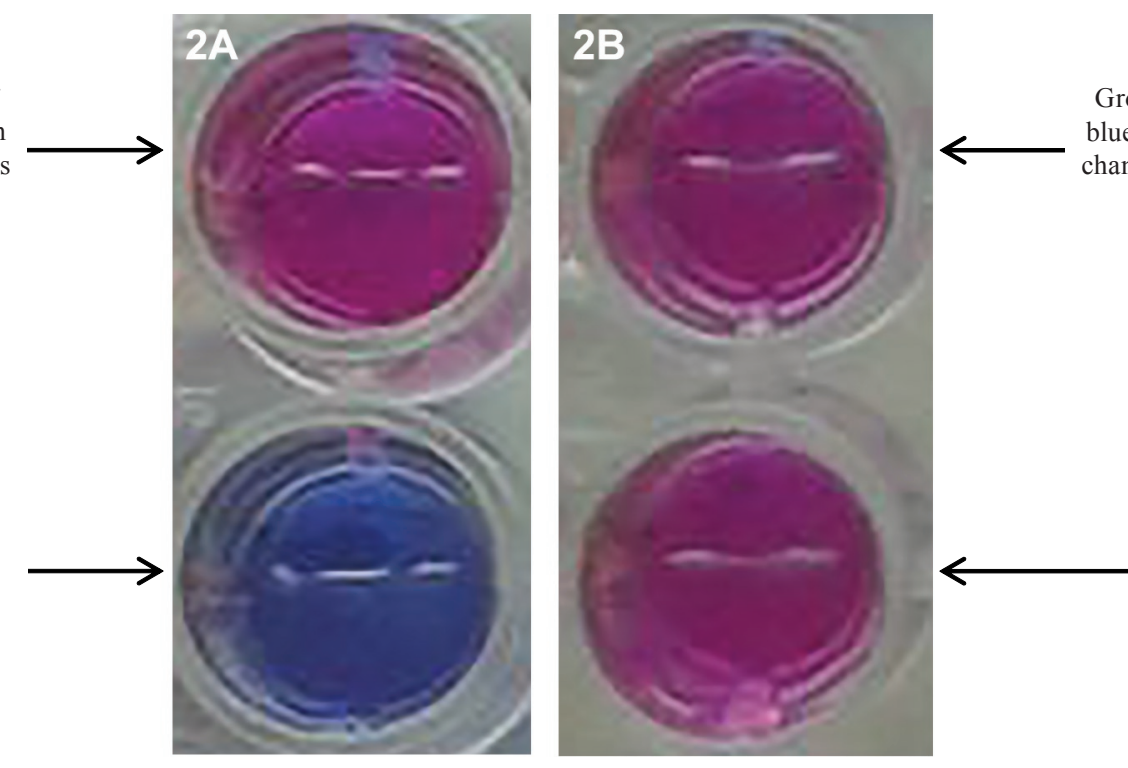

Growth control well. If blue colour of resazurin changes to pink, there is bacterial growth.

Antibiotic containing well. There is bacterial growth, resistant isolate.

Fig. 2: resazurin microtitre assay.

Growth control well. If the malachite green decolourised, there is bacterial growth.

Antibiotic containing well. There is no any bacterial growth, susceptible isolate.

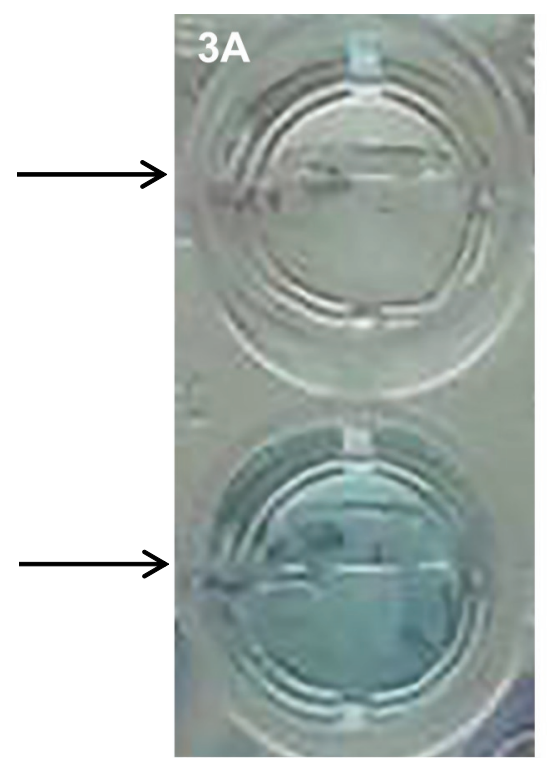

Fig. 3: malachite green decolourisation assay.

\section{RESULTS}

All data are summarised in Table. The specificity, sensitivity, PPV, NPV and agreement of the MNRA for INH were $93.3 \%, 100 \%, 90.9 \%, 100 \%$ and $96 \%$, respectively. Two isolates were susceptible to INH by the reference method, but resistant by the MNRA. The values for RIF were $90.9 \%, 100 \%, 85 \%, 100 \%$ and $94 \%$, respectively. Three isolates were shown to be susceptible to RIF by the reference method, whereas they were resistant according to the MNRA.

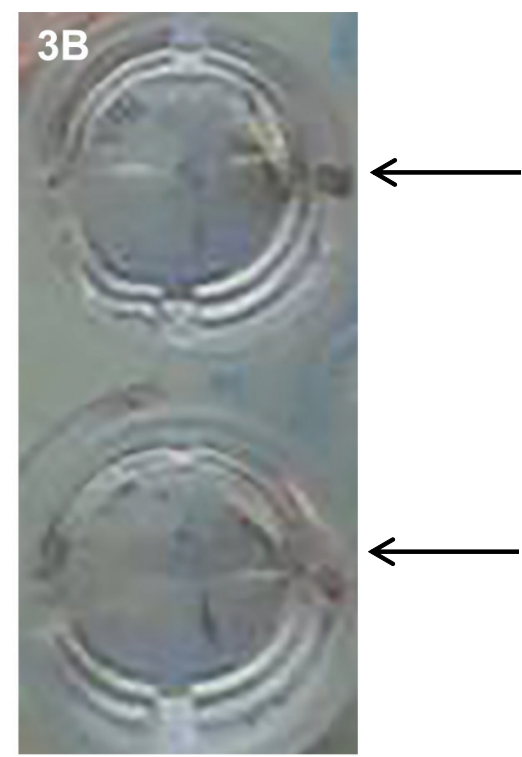

Growth control well. If the malachite green decolourised, there is bacterial growth.

Antibiotic containing well. There is bacterial growth, resistant isolate.
The specificity, sensitivity, PPV, NPV and agreement of the REMA for INH were 100\%, 95\%, 100\%, 96.7\% and $98 \%$, respectively. One isolate was susceptible to INH by the REMA but resistant by the reference method. These values for RIF were 100\%, 94.1\%, 100\%, 97\% and $98 \%$, respectively. One isolate was resistant to RIF by the reference method but susceptible by the REMA.

The specificity, sensitivity, PPV, NPV and agreement of the MGDA for INH were $100 \%, 95 \%, 100 \%, 96.7 \%$ and $98 \%$, respectively. One isolate was susceptible to INH 
Growth control well. Blue/purple colour of crystal violet is decolorised, there is bacterial growth.
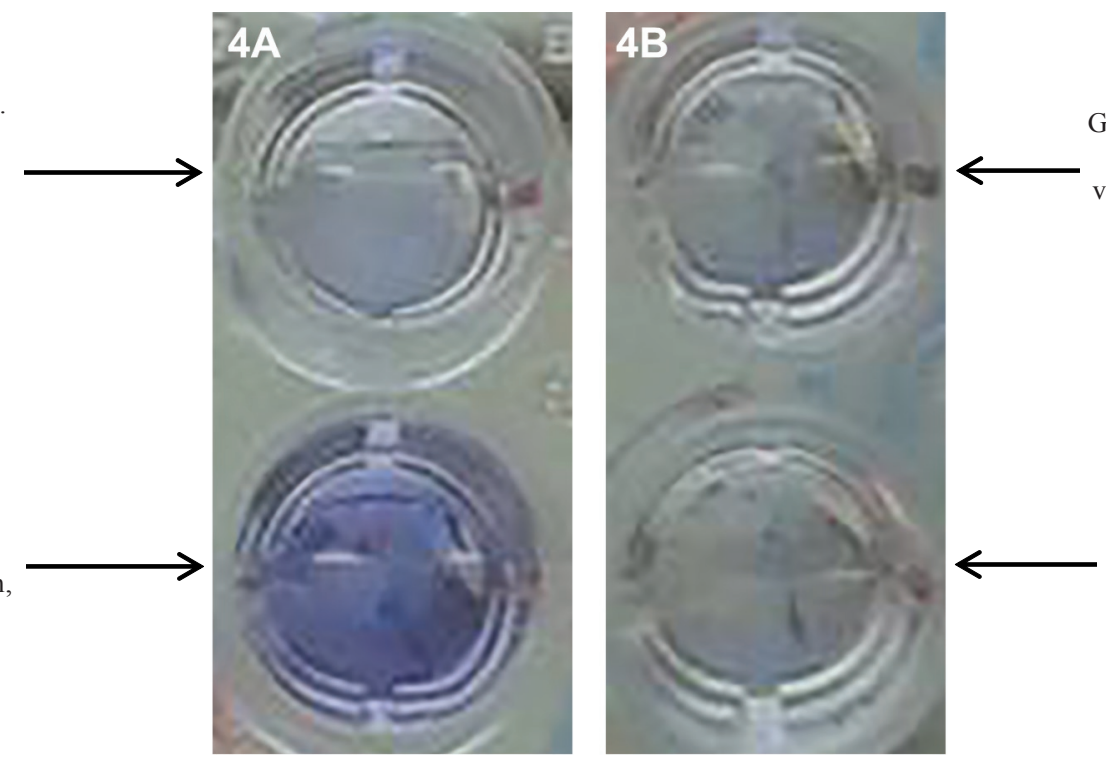

Growth control well. Blue/ purple colour of crystal violet is decolorised, there is bacterial growth.

Antibiotic containing well. There is bacterial growth, resistant isolate.

Fig. 4: crystal violet decolourisation assay.

TABLE

Comparing the results of colourimetric methods with those obtained with reference method

\begin{tabular}{|c|c|c|c|c|c|c|c|c|}
\hline \multirow{2}{*}{$\begin{array}{l}\text { Drug } \\
(\%)\end{array}$} & \multirow[b]{2}{*}{ MNRA } & \multicolumn{2}{|c|}{ Reference method ${ }^{a}$} & \multirow{2}{*}{$\begin{array}{c}\text { Specificity } \\
(\%)\end{array}$} & \multirow{2}{*}{$\begin{array}{c}\text { Sensitivity } \\
(\%)\end{array}$} & \multirow{2}{*}{$\begin{array}{l}\text { PPV } \\
(\%)\end{array}$} & \multirow{2}{*}{$\begin{array}{c}\text { NPV } \\
(\%)\end{array}$} & \multirow{2}{*}{$\begin{array}{c}\text { Agreement } \\
(\%)\end{array}$} \\
\hline & & $\mathrm{R}$ & $\mathrm{S}$ & & & & & \\
\hline INH & $\mathrm{R}$ & 20 & 2 & 93.3 & 100 & 90.9 & 100 & 96 \\
\hline 96 & $\mathrm{~S}$ & 0 & 28 & & & & & \\
\hline RIF & $\mathrm{R}$ & 17 & 3 & 90.9 & 100 & 85 & 100 & 94 \\
\hline 94 & $\mathrm{~S}$ & 0 & 30 & & & & & \\
\hline
\end{tabular}

REMA

$\begin{array}{lcccccccc}\text { INH } & \text { R } & 19 & 0 & 100 & 95 & 100 & 96.7 & 98 \\ 98 & \mathrm{~S} & 1 & 30 & & & & & 97 \\ \text { RIF } & \mathrm{R} & 16 & 0 & 100 & 94.1 & 100 & 98 \\ 98 & \mathrm{~S} & 1 & 33 & & & & \end{array}$

\section{MGDA}

$\begin{array}{lcccccccc}\text { INH } & \text { R } & 19 & 0 & 100 & 95 & 100 & 96.7 & 98 \\ 98 & \text { S } & 1 & 30 & & & & & 97 \\ \text { RIF } & \text { R } & 16 & 0 & 100 & 94.1 & 100 & 98 \\ 98 & \text { S } & 1 & 33 & & & & \end{array}$

\section{CVDA}

$\begin{array}{lcccccccc}\text { INH } & \text { R } & 19 & 0 & 100 & 95 & 100 & 96.7 & 98 \\ 98 & \mathrm{~S} & 1 & 30 & & & & & 97 \\ \text { RIF } & \mathrm{R} & 16 & 0 & 100 & 94.1 & 100 & 98 \\ 98 & \mathrm{~S} & 1 & 33 & & & & & \end{array}$

a: agar proportion method on 7H11 agar; CVDA: crystal violet decolourisation assay; INH: isoniazid; MGDA: malachite green decolourisation assay; MNRA: microtitre nitrate reductase assay; NPV: negative predictive value; PPV: positive predictive value; R: resistant; REMA: resazurin microtitre assay; RIF: rifampicin; S: susceptible. 
by the MGDA, but resistant by the reference method. The values for RIF were $100 \%, 94.1 \%, 100 \%, 97 \%$ and $98 \%$, respectively. One isolate was resistant to RIF by the reference method, but susceptible by the MGDA test.

The specificity, sensitivity, PPV, NPV and agreement of the CVDA for INH were $100 \%, 95 \%, 100 \%$, $96.7 \%$ and $98 \%$, respectively. One isolate was susceptible to INH by the CVDA, but resistant by the reference method. For RIF, these values were $100 \%, 94.1 \%, 100 \%$, $97 \%$ and $98 \%$, respectively. One isolate was shown to be resistant to RIF by the reference method, whereas it was susceptible according to the CVDA.

The MNRA results were reported immediately after Griess reagent was added on the seventh day of the incubation, while the results of the REMA, MGDA and CVDA methods were reported on the eighth-10th day after inoculation due to the one-three days of incubation that were required.

\section{DISCUSSION}

For susceptibility testing of M. tuberculosis isolates, the use of colourimetric methods has increased in recent years because these methods are easy to perform, reproducible, reliable, readily available and very low in cost. In this study, colourimetric methods, including the MGDA, MNRA, REMA and CVDA, were compared with each other in terms of sensitivity, specificity, PPV and NPV.

In this study, the agreement of the MNRA was $96 \%$ for INH and $94 \%$ for RIF. In the study performed by Coban et al. (2012), the rates for both anti-TB drugs were found to be $100 \%$ for the NRA. In another study, Montoro et al. (2005) reported that the sensitivity and specificity of the NRA for INH and RIF were $95.6 \%$ and $100 \%$, respectively.

Agreements for the REMA were detected to be $98 \%$ for both drugs. One study showed that the sensitivities, specificities, PPVs, NPVs and agreements of INH and RIF for the REMA were 100-91.3\%, 90.4-100\%, 88.5$100 \%, 100-96.1 \%$ and $94.5-97.2 \%$, respectively (Coban et al. 2012). Nour et al. (2013) also reported that sensitivities, specificities, PPVs, NPVs and agreements of INH and RIF were $100 \%$ by this method.

In the current study, the agreements for both drugs were $98 \%$. Farnia et al. (2008) reported that both INH and RIF sensitivities, specificities and agreements were $100 \%$. In another study, Coban and Uzun (2013) reported that the sensitivity, specificity, PPV, NPV and agreement of the MGDA for INH and RIF were 100-95.6\%, 97.6-100\%, $96.8-100 \%, 100-98 \%$ and $98.6-98.6 \%$, respectively.

In this study, agreements were detected as $98 \%$ for both drugs by the CVDA. Earlier studies performed by Coban (2014) found that the rates for INH and RIF were $92.5-88.8 \%, 96.4-100 \%, 96.1-100 \%, 93.1-94.8 \%$ and $94.5-96.3 \%$, respectively.

The specificity of the MNRA test was found to be lower than that of the other tests because the reference method yielded sensitive results for antibiotics whereas the MNRA test yielded resistant results (2 isolates for INH and 3 isolates for RIF). NRA is usually performed on LJ medium, but it was performed in liquid medium in this study. A false colour change may occur because following the addition of $\mathrm{KNO}_{3}$ and drugs to the liquid medium, viable bacteria reduce $\mathrm{KNO}_{3}$ to nitrite until they are killed by the antibiotic. This change may cause a method to define susceptible isolates as resistant.

In the MNRA, the time required for detection is less than for the other colourimetric methods and this test does not require additional incubation time, but the Griess reagent, which consists of three chemical substances, should be freshly prepared. The REMA requires resazurin powder that should be freshly prepared and this method requires additional incubation time. The MGDA and CVDA have similar characteristics to the REMA, but MG and CV dyes are obtained easily. Moreover, CV (used for Gram staining) is available in clinical microbiology laboratories. Evaluating all of the colourimetric methods, each has some advantages and disadvantages. Nonetheless, all of the above methods are reliable, easy to perform, cheap and easy to evaluate and do not require special equipment. In conclusion, these methods may be used in mycobacteriology laboratories in both developed and developing countries.

\section{REFERENCES}

Angeby KA, Klintz L, Hoffner SE 2002. Rapid and inexpensive drug susceptibility testing of Mycobacterium tuberculosis with a nitrate reductase assay. $J$ Clin Microbiol 40: 553-555.

Bwanga F, Hoffner S, Haile M, Joloba ML 2009. Direct susceptibility testing for multidrug resistant tuberculosis: a meta-analysis. BMC Infect Dis 9: 67.

Chang K, Lu W, Wang J, Zhang K, Jia S, Li F, Deng S, Chen M 2012. Rapid and effective diagnosis of tuberculosis and rifampicin resistance with Xpert MTB/RIF assay: a meta-analysis. $J$ Infect 64: $580-588$.

Coban AY 2014. A new rapid colourimetric method for testing $M y$ cobacterium tuberculosis susceptibility to isoniazid and rifampicin: a crystal violet decolourisation assay. Mem Inst Oswaldo Cruz 109: 246-249.

Coban AY, Birinci A, Ekinci B, Durupinar B 2004. Drug susceptibility testing of Mycobacterium tuberculosis by the broth microdilution method with 7H9 broth. Mem Inst Oswaldo Cruz 99: 111-113.

Coban AY, Uzun M 2013. Rapid detection of multidrug-resistant Mycobacterium tuberculosis using the malachite green decolourisation assay. Mem Inst Oswaldo Cruz 108: 1021-1023.

Coban AY, Uzun M, Akgunes A, Durupinar B 2012. Comparative evaluation of the microplate nitrate reductase assay and the rezasurin microtitre assay for the rapid detection of multidrug resistant Mycobacterium tuberculosis clinical isolates. Mem Inst Oswaldo Cruz 107: 578-581.

Farnia P, Masjedi MR, Mohammadi F, Tabarsei P, Farnia P, Mohammadzadeh AR, Baghei P, Varahram M, Hoffner S, Velayati AA 2008. Colorimetric detection of multidrug-resistant or extensively drug-resistant tuberculosis by use of malachite green indicator dye. J Clin Microbiol 46: 796-799.

Friedrich SO, Venter A, Kayigire XA, Dawson R, Donald PR, Diacon AH 2011. Suitability of Xpert MTB/RIF and Genotype MTBDRplus for patient selection for a tuberculosis clinical trial. $J$ Clin Microbiol 49: 2827-2831.

Kent PT, Kubica GP 1985. Public health mycobacteriology: a guide for the level III laboratory, US Department of Health and Human Services, Atlanta, $207 \mathrm{pp}$. 
Martin A, Paasch F, Docx S, Fissette K, Imperiale B, Ribón W, González LA, Werngren J, Engström A, Skenders G, Juréen P, Hoffner S, Del Portillo P, Morcillo N, Palomino JC 2011. Multicentre laboratory validation of the colorimetric redox indicator (CRI) assay for the rapid detection of extensively drug-resistant (XDR) Mycobacterium tuberculosis. J Antimicrob Chemother 66: 827-833.

Martin A, Panaiotov S, Portaels F, Hoffner S, Palomino JC, Angeby K 2008. The nitrate reductase assay for the rapid detection of isoniazid and rifampicin resistance in Mycobacterium tuberculosis: a systematic review and meta-analysis. J Antimicrob Chemother 62: 56-64.

Martin A, Portaels F, Palomino JC 2007. Colorimetric redox-indicator methods for the rapid detection of multidrug resistance in $\mathrm{Myco}$ bacterium tuberculosis: a systematic review and meta-analysis. $J$ Antimicrob Chemother 59: 175-183.
Montoro E, Lemus D, Echemendia M, Martin A, Portaels F, Palomino JC 2005. Comparative evaluation of the nitrate reductase assay, the MTT test and the resazurin microtiter assay for drug susceptibility testing of clinical isolates of Mycobacterium tuberculosis. $J$ Antimicrob Chemother 55: 500-505.

Nour MS, El-Shokry MH, Shehata IH, Aziz AMA-E 2013. Evaluation of rezasurin microtiter assay and high resolution melting curve analysis for detection of rifampicin and isoniazid resistance of $M y$ cobacterium tuberculosis clinical isolates. Clin Lab 59: 763-771.

Palomino JC, Martin A, Portaels F 2007. Rapid drug resistance detection in Mycobacterium tuberculosis: a review of colourimetric methods. Clin Microbiol Infect 13: 754-762.

WHO - World Health Organization 2014. Global tuberculosis report 2014. Available from: apps.who.int/iris/bitstream/10665/137094/ 1/9789241564809 eng.pdf?ua=1. 